\title{
Repressor Element-1 Silencing Transcription/Neuron-Restrictive Silencer Factor Is Required for Neural Sodium Channel Expression during Development of Xenopus
}

\author{
Ricardo Armisén, ${ }^{*}$ Rómulo Fuentes, ${ }^{*}$ Patricio Olguín, María E. Cabrejos, and Manuel Kukuljan \\ Programa de Fisiología y Biofísica, Instituto de Ciencias Biomédicas, Facultad de Medicina, Universidad de Chile, \\ Independencia 1027, Santiago, Chile
}

The ability of neurons to fire rapid action potential relies on the expression of voltage-gated sodium channels; the onset of the transcription of genes that encode these channels occurs during early neuronal development. The factors that direct and regulate the specific expression of ion channels are not well understood. Repressor element-1 silencing transcription/ neuron-restrictive silencer factor (REST/NRSF) is a transcriptional regulator characterized as a repressor of the expression of NaV1.2, the gene encoding the voltage-gated sodium channel most abundantly expressed in the CNS, as well as of the expression of numerous other neuronal genes. In mammals, REST/NRSF is expressed mostly in non-neural cell types and immature neurons, and it is downregulated on neural maturation. To understand the mechanisms that govern sodium channel gene transcription and to explore the role of REST/NRSF in vivo, we inhibited REST/NRSF action in developing Xenopus

The generation of rapid action potentials relies on the adequate expression of particular voltage-gated ion channels. During embryonic development of Xenopus laevis, primary spinal neurons transit from an electrically silent state to a condition in which they can fire mature neuronal action potentials (Spitzer and Lamborghini, 1976). Underlying this transition is the onset of the expression of voltage-gated channels (O'Dowd et al., 1988; Desarmenien et al., 1993; Olson, 1996), particularly the beginning of the expression of voltage-gated sodium currents and the rapid increase in the density of potassium currents. This process is inhibited by blockers of RNA synthesis, indicating that activation of transcription is fundamental in the acquisition of electrical activity in neurons (Ribera and Spitzer, 1989). The identity of the potassium channels upregulated during these stages has been unveiled (Ribera, 1990; Burger and Ribera, 1996; Gurantz et al., 1996; Vincent et al., 2000), but the mechanisms that govern the transcription of ion channels are unknown.

\footnotetext{
Received April 11, 2002; revised June 14, 2002; accepted July 22, 2002.

This work was supported by Fondo Nacional de Desarrollo Científico y Tecnológico 2980063, 4000031, 1961084, and 1000824; Fundación G. Puelma; fellowships from The Company of Biologists Ltd. and Fundación de Estudios Biomédicos Avanzados; and a graduate Comisión Nacional de Deserollo Científico y Tecnológico Scholarship (R.A.). We thank Dr. G. Mandel and J. Sierralta for continuous support, A. Figueroa and F. Vergara for excellent technical assistance, and Dr. M. E. Andrés for comments on this manuscript.

*R.A. and R.F. contributed equally to this work.

Correspondence should be addressed to Manuel Kukuljan, Programa de Fisiología y Biofísica, Instituto de Ciencias Biomédicas, Facultad de Medicina, Universidad de Chile, Independencia 1027, Santiago, Chile. E-mail: kukuljan@neuro.med.uchile.cl. Copyright (C) 2002 Society for Neuroscience $0270-6474 / 02 / 228347-05 \$ 15.00 / 0$
}

laevis embryos by means of a dominant negative protein or antisense oligonucleotides. Contrary to what was expected, these maneuvers result in the decrease of the expression of the NaV1.2 gene, as well as of other neuronal genes in the primary spinal neurons and cranial ganglia, without overt perturbation of neurogenesis. These results, together with the demonstration of robust REST/NRSF expression in primary spinal neurons, suggest that REST/NRSF is required for the acquisition of the differentiated functional neuronal phenotype during early development. Furthermore, they suggest that REST/NRSF may be used to activate or repress transcription of neuronal genes in distinct cellular and developmental contexts.

Key words: primary spinal neurons; neuronal differentiation; repressor element silencer of transcription; dominant negative; antisense oligonucleotides; sodium channels
Repressor element silencing transcription/neuron-restrictive silencer factor (REST/NRSF) was identified as a factor that influences the expression of voltage-gated sodium channels (Chong et al., 1995) and also as a regulator of the expression of other genes (Schoenherr and Anderson, 1995). REST/NRSF binds a DNA element (RE-1) contained in the regulatory regions of numerous neuronal genes, including NaV1.2 (Kraner et al., 1992; Mori et al., 1992; Schoenherr et al., 1996). The extensive characterization of REST/NRSF has led to the current view of this protein as a repressor of neural-specific gene expression; REST/NRSF is expressed in non-neural cells and undifferentiated neurons and downregulated on differentiation. REST/NRSF interacts with the corepressors Sin3 and CoREST, assembling complexes that recruit histone deacetylase activity to regions of genes that bear the RE-1 sequence (Andrés et al., 1999; Huang et al., 1999; Naruse et al., 1999; Grimes et al., 2000; Ballas et al., 2001). The in vivo role of REST/NRSF has been difficult to ascertain, given the early embryonic lethality of mice bearing a homozygous deletion of the REST/NRSF gene (Chen et al., 1998). Expression of the DNAbinding domain (DBD) of REST/NRSF in chicken embryos associates with derepression of neural genes (Chen et al., 1998), whereas constitutive expression of the murine isoform in developing neurons associates with repression of some neuronal genes and defects in axon guidance (Paquette et al., 2000). Some studies have found the expression of REST/NRSF mRNA in adult neurons (Palm et al., 1998); furthermore, it has been shown that it can act as a transcriptional activator for some genes (Bessis et al., 
1997; Kallunki et al., 1998; Seth and Majzoub, 2001). Some reports also indicate that REST/NRSF may be involved in the regulation of the expression of non-neural genes (Scholl et al., 1996; Kuwahara et al., 2001). These data suggest that the actual function of REST/NRSF may be more complex than originally envisioned and that it could vary according to the cellular context in which it is studied.

\section{MATERIALS AND METHODS}

$x R E S T-D B D$ construction. The DNA-binding domain of xREST (GenBank accession AF096301), comprising the sequence that encodes K138 to Q440, was amplified by PCR with the primers GCGGAATTCAAAAAGGACACCCAGCCCAAC and CGCCTCGAGTCATTGCTTATTTGCATCAACATCTG, adding one EcoRI and one XhoI restriction sites (underlined in the primers sequences). The PCR product was cloned into the plasmid CS2+NLSMT, kindly provided by Dr. D. Turner (University of Michigan, Ann Arbor, MI). This vector adds a nuclear localization signal and six Myc epitopes to the N-terminal coding region.

Embryo manipulation, injection, and in situ hybridization. X. laevis embryos were generated by in vitro fertilization, dejellied, and incubated following standard procedures. A portion $(0.5-2 \mathrm{ng})$ of in vitro transcribed xREST-DBD RNA or 2-20 ng of a morpholino-modified antisense oligonucleotide (sequence ACTGGTTGACCATTTGAGTGGC) (Gene Tools, Philomath, OR) were injected in a total volume of $10 \mathrm{nl}$, in one cell at the two cell stage or in two cells at the four cell stage. The RNA encoding $\beta$-galactosidase $(0.5 \mathrm{ng})$ was coinjected in all cases. Embryos were incubated at $18^{\circ} \mathrm{C}$ until neurula stage and then prefixed; the injected side was detected by developing the $\beta$-galactosidase reaction, and the whole-mount in situ hybridization (ISH) procedure, using 11UTP digoxigenin (Boehringer Mannheim, Mannheim, Germany)-labeled probes, was followed according to described methods (Sive et al., 2000).

ISH probes. $x N a V 1.2$ was generated by reverse transcription-PCR (RT$\mathrm{PCR}$ ) using primers designed on the basis of the conserved sequences in vertebrate homologs and RNA isolated from $X$. laevis neural tube as input. The primers sequence was CCIRYIAAYGGIAARATGA and GGIGCIYTNGCNTTYGARGAY. PCR fragments were cloned (Topo-TA; Invitrogen, Carlsbad, CA) and sequenced (GenBank access code AY121368). The $X$. laevis SCG10 (GenBank X71434) probe was also generated by RT-PCR with the primers GCTCGCTACCCTCTCCACT and CATACTGATATCGCATGATCCGT. Other probes were obtained from the sources indicated: Xslug, $\mathrm{Xsox} 2$, and N- $\beta$ II tubulin from Roberto Mayor (Universidad de Chile, Santiago, Chile); Hox11L2 from Paul Krieg (University of Arizona Health Sciences Center, Tucson, AZ); Pax3 from Andre Brandli (Swiss Federal Institute of Technology, Zurich, Switzerland); and xREST8 from Gail Mandel (Howard Hughes Medical Institute, State University of New York, Stony Brook, NY).

$R T-P C R$, cell culture, and single-cell RT-PCR. RT-PCR detection of the REST/NRSF transcript was performed using total RNA isolated from whole embryos or dissected tissues and the following primers: F1, AGAACGACCATATCAGTGTATTC; R2, CTGAGTAGATGACAAGCGCC; R1, AATCAGATTTTCTTTTCACACAACTAGA.

Neural plate cell cultures were prepared, and subsequent single-cell RT-PCR of morphologically differentiated neurons was conducted as described (Ribera and Spitzer, 1989; Gurantz et al., 1996). Two different nested PCR reactions were performed with the samples obtained from one cell, one for neuronal tubulin (N-tubulin) mRNA (231 bp product) and the other for xREST/NRSF. The primers for N-tubulin were as follows: F1, GCCAGTGCGGTAACCAAATTG; F2, ACACGGCATTGATCCTACAG; R2, AGCTCCTTCGGTGTAATGAC; R1, TCATGATTCGGTCTGGGTACTC.

To discard contamination with genomic DNA, xREST/NRSF primers were designed flanking an intron; amplification of genomic DNA results in fragments of $\sim 2 \mathrm{~kb}$, in contrast with the 557 and $660 \mathrm{bp}$ fragments resulting from the amplification of cDNA. PCR products were cloned and sequenced to confirm their identities.

Electrophoretical mobility shift assays. Gel retardation assays were performed using $0.1-1 \mathrm{ng}$ of a 3 '-end ${ }^{32} \mathrm{P}$-labeled DNA fragment containing two RE-1 motifs in tandem, kindly provided by Dr. G. Mandel (Chong et al., 1995). Crude protein extracts were prepared by freon extraction of uninjected or injected embryos homogenized in the presence of a mixture of protease inhibitors. The protein extracts and the antibody against the Myc epitope (monoclonal antibody 9E10; Sigma, St. Louis, MO) were added to the mix and incubated at $4^{\circ} \mathrm{C}$ for $15 \mathrm{~min}$. Then, the labeled
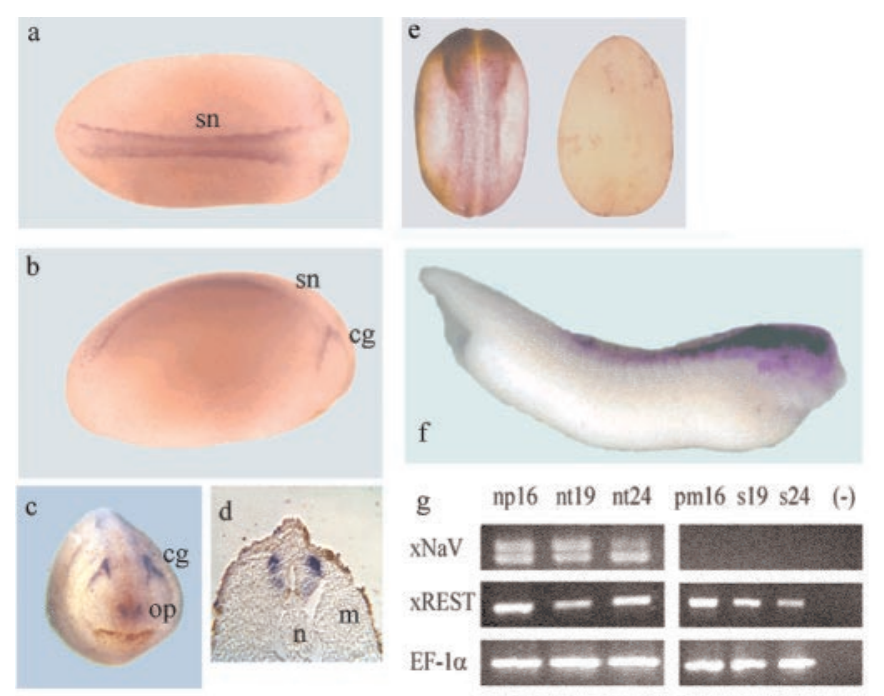

Figure 1. NaV1.2 and REST/NRSF are expressed in neural tissues during development of $X$. laevis. $a-d$, ISH for xNaV1.2: expression is restricted to primary spinal neurons $(s n)$, cranial ganglia primordia $(c g)$, and olfactory placodes $(o p)$. In a section of a stage 18 embryo, $x N a V 1.2$ expression is observed in the lateral and ventral regions of the neural tube. $a$, Dorsal view, stage 18 ; $b$, lateral view, stage 18 ; $c$, anterior view, stage 24; $d$, central transversal section, stage 18 ; $n$, notochord; $m$, presomitic mesoderm; $e$, left, ISH showing diff use expression of REST during neurulation (stage 18), including neural folds; right, a sense REST probe does not produce significant labeling in a stage 18 embryo; $f$, at stage 35 , REST/ NRSF expression is stronger in the anterior neural tissue; $g$, RT-PCR showing the coexpression of xNaV1.2 and REST/NRSF in dissected tissues at the stages annotated; $n p$, neural plate; $n t$, neural tube; $p m$, presomitic mesoderm; $s$, somites. The constitutively expressed transcript $\mathrm{EF} 1 \alpha$ is shown as a control.

probe was added, and the reactions were incubated at $30^{\circ} \mathrm{C}$ for $25 \mathrm{~min}$. The resulting complexes were analyzed in $4 \%$ polyacrylamide gels containing $5 \%$ glycerol and $0.5 \times$ Tris-borate-EDTA buffer. The gels were dried, and the complexes were visualized by autoradiography.

\section{RESULTS}

\section{REST and NaV1.2 are expressed in primary neurons during neurulation}

To study the regulation of the expression of sodium channel genes by REST/NRSF during neurulation, we generated a probe for the $X$. laevis $\mathrm{NaV} 1.2$ ortholog $(\mathrm{xNaV} 1.2)$. $\mathrm{xNaV} 1.2$ is expressed from stage 16 in neural plate, as evaluated by RT-PCR, whereas ISH detects transcripts from stage 18 in spinal neurons, which is consistent with functional studies of the onset of sodium current expression in this system (Fig. $1 a-d, g$ ). Analysis of sections shows the expression of this transcript restricted to the neural tube, particularly to its lateral and ventral regions (Fig. 1d). At later stages, $\mathrm{xNaV1.2}$ is also expressed in the cranial ganglia primordia (Fig. 1c).

We then examined the expression pattern of REST/NRSF. $X$. laevis REST/NRSF mRNA is widely expressed in the developing embryo; at the neurula stage, it is found in the neural folds and neural tube, paraxial mesoderm, and other structures, as revealed by ISH and RT-PCR using RNA obtained from dissected tissues as input (Fig. 1e,g). It is of interest that during neurulation, the transcripts encoding $\mathrm{xREST} / \mathrm{NRSF}$ and $\mathrm{xNaV1.2}$ are coexpressed in developing neural tissues. At later stages of development, during differentiation of secondary neurons (stage 35), xREST/ NRSF expression is prominent in the nervous system (Fig. 1f). To confirm that the expression of REST/NRSF detected by ISH 


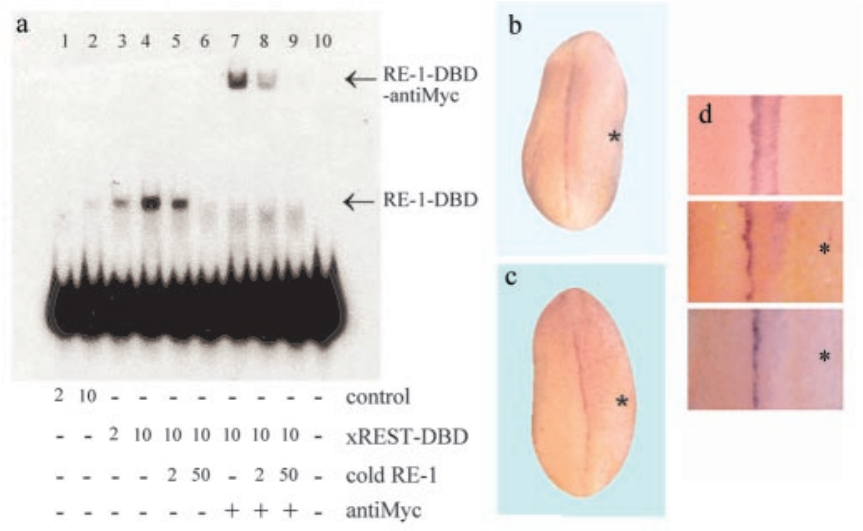

Figure 2. DBD-xREST binds specifically to the RE-1 and inhibits $\mathrm{xNaV1.2}$ expression. $a$, Electrophoretical mobility shift assay analysis of the complex formation by the use of protein extracts of noninjected embryos (lanes 1-2) and embryos injected with the DBD-xREST RNA (lanes 3-9). Two or $10 \mu \mathrm{g}$ of total protein was used in each assay. DBD-xREST binds to RE-1 (lanes 3-6). The addition of antibody against the Myc epitope produces the super-retardation of the complex (lanes 7-9). The binding specificity of the complex was evaluated by the addition of 2- or 50-fold excess of cold probe (lanes 5-6, 8-9). The probe alone is shown in Lane 10. Arrows indicate the complexes. $b$, Expression of xRESTDBD inhibits $x N a V 1.2$ expression during neurulation. ISH for $x N a V 1.2$; the injected side is marked by an asterisk. Notice a single line of $\mathrm{xNaV1.2}$ labeling, in contrast with Figure 1a. c, Injection of a morpholino-modified antisense oligonucleotide targeting xREST/NRSF inhibits xNaV 1.2 expression at the time of neurulation. $d$, Detail of a noninjected embryo (top panel) and embryos injected with xREST-DBD RNA (middle panel) or the antisense oligonucleotide (bottom panel) in one of two cells.

actually occurs in neurons, we analyzed its presence in identified mature neurons in culture by single-cell RT-PCR. These neurons display fully differentiated functional and morphological phenotype. In 6 of 14 cells positively identified as neurons both by morphology and by the expression of the transcript encoding neuronal tubulin, we detected the presence of REST/NRSF transcripts (data not shown).

\section{Inhibition of REST/NRSF leads to decreased expression of NaV1.2 and other neuronal genes}

We constructed a mutated version of $X$. laevis $\mathrm{REST} / \mathrm{NRSF}$ cDNA, which encodes a protein devoid of the transrepressor domains described for the mammalian ortholog. This protein comprises only the DBD (xREST-DBD), which shows an identity of $85 \%$ with the human and murine orthologs, including the amino acids that are critical for binding RE-1. According to the biochemical characterization of REST/NRSF, this domain can compete with the endogenous REST/NRSF for the RE-1 binding sequence, antagonizing the transcriptional effects of the wild-type protein (Chong et al., 1995). Injection of the in vitro-transcribed xREST-DBD RNA into embryos at the one or two cell stages resulted in the expression of the recombinant protein throughout early development and included neurulation, as recognized by Western analysis (data not shown). We then assayed the ability of the expressed xREST-DBD protein to bind RE-1. As shown in Figure $2 a$, crude extracts prepared from stage 18 embryos injected with the xREST-DBD RNA contain a marked RE-1 binding activity. Extracts from uninjected embryos also appear to contain this activity, although in a much lesser amount (Lane 2). The supershift induced by the incubation with the anti-Myc antibody indicates that the observed RE- 1 binding activity is because of the expressed recombinant form.
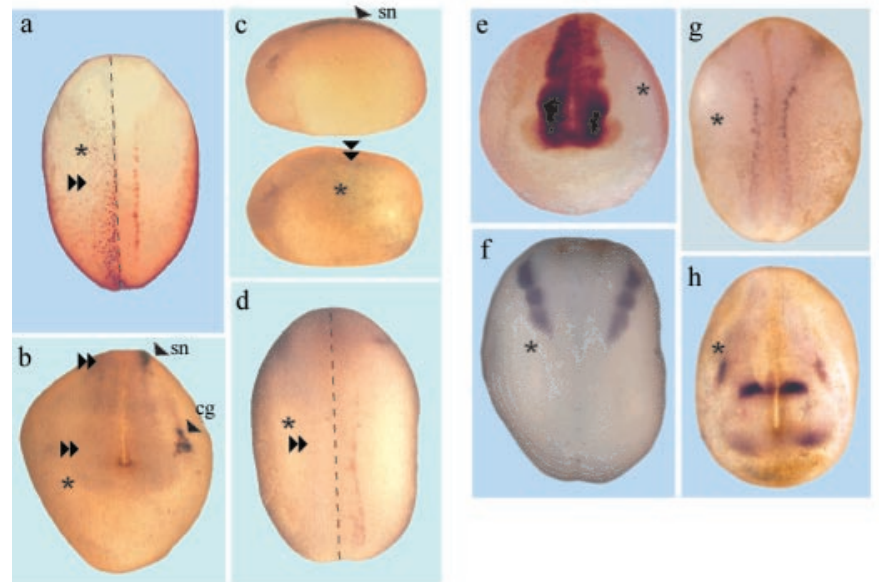

Figure 3. xREST-DBD decreases the expression of other neuronal genes but does not perturb neural induction. $a-c$, ISH for N-tubulin; the injected side is marked by an asterisk; $b$, both lateral views; $c$, anterior view of the same embryo, where the arrowheads signal the label of the primary spinal neurons $(s n)$ and cranial ganglia $(c g) . d$, xREST-DBD represses SCG10 expression. Double arrowheads signal the expected normal position of $\mathrm{N}$-tubulin or SCG10 expression. The spotted labeling in the injected side (a) and the diffuse hue in $c$ result from $\beta$-galactosidase activity staining. $e-h$, xREST-DBD does not affect neural induction and early differentiation. Expression patterns of Sox2 $(e)$, Slug $(f)$, Hox11L12 $(g)$, and Pax-3 (h). $e, h$, Anterior views; $f, g$, dorsal views.

Expression of xREST-DBD resulted in a significant decrease of the expression of the $x \mathrm{NaV} 1.2$ RNA in the spinal territory of the injected side; this effect was observed in $49 \%$ of the studied embryos ( $n=105$; three experiments). We did not observe ectopic expression of $\mathrm{xNaV} 1.2$ or increased normotopic expression, as compared with the uninjected side (Fig. $2 b$, middle panel in $d$ ). To test in a mechanistically independent manner the functional role of REST/NRSF in vivo, we injected a morpholinomodified antisense oligonucleotide against the xREST/NRSF into one cell at the two-cell stage embryos and examined $\mathrm{xNaV} 1.2$ expression at the neural tube stage. We observed a dosedependent decrease of the transcript encoding this sodium channel in spinal neurons; at the maximal dose of the oligonucleotide tested (20 ng/injection), $50 \%$ of the embryos displayed defects in the expression of $\mathrm{xNaV} 1.2$ ( $n=130$; three experiments); we did not observe extraneural expression of this transcript (Fig. $2 c$, bottom panel in $d$ ).

Because many genes contain RE-1 sequences in their regulatory regions, we assayed the effect of xREST-DBD on the expression of other neuronal-specific genes known to contain RE-1 sequences in other species. We found a consistent decrease in the expression of the N-tubulin and SCG10 transcripts in the primary neural territories. Thus, $58 \%$ of the embryos injected with the xREST-DBD RNA displayed partial or complete absence of the $\mathrm{N}$-tubulin transcript in the injected side $(n=112$; four experiments), whereas absence of SCG10 was observed in $61 \%$ of the cases ( $n=98$; three experiments) (Fig. $3 a-d)$.

\section{Inhibition of REST function does not affect neurogenesis}

The decreased expression of NaV1.2, N-tubulin, and SCG10 associated to the perturbation of xREST/NRSF function may be explained by decreased transcription of these neural-specific genes in a committed neuron as well as by a failure in neuronal differentiation, leading to the lack of neurons able to express 
these transcripts. To differentiate between these alternatives, we evaluated the effect of xREST-DBD on the expression patterns of genes used as markers of neural induction and differentiation. We did not find significant perturbations in the patterns of expression of the neural plate marker XSox2, the neural crest marker xSlug, and the homeobox genes Hox11L2 and Pax-3, which are expressed in Rohon-Beard and a subset of spinal interneurons, respectively (Fig. $3 e-h)$.

\section{DISCUSSION}

To advance our understanding of the molecular mechanisms that underlie the concerted and specific expression of ion channels, we considered the detailed physiological characterization of the development of excitability performed in Xenopus primary spinal neurons. Although conservation of general mechanisms through phylogeny is expected, comparison of our results with those obtained in mammalians must consider that in amphibians, a subpopulation of neurons (primary neurons) withdraws from the mitotic cycle during early embryogenesis and forms a circuit that controls early larval behavior. In Xenopus embryos, primary neurons undergo their last round of division during neurulation (around stage 15) and then differentiate (Hartenstein, 1989). Our study focuses exclusively on this population. At the time at which we examine expression of $\mathrm{xNaV} 1.2$ transcripts, primary spinal neurons are in final stages of their differentiation, although the embryo is still undergoing developmental processes previous to organogenesis. In contrast, secondary neuron precursors continue replicating until stage 35 or later. Much less is known about the physiological development of this population.

We explored the role of REST/NRSF in the onset of the expression of $x \mathrm{NaV} 1.2$. Considering the current view about REST/NRSF function, and the widespread expression of xREST/ NRSF before the onset of the expression of $x \mathrm{NaV1.2}$, we postulated that a decrease in the expression of REST at the time of primary neuron differentiation would allow the function of activators of the transcription of this and other neural genes. However, we observed a maintained expression of the REST/NRSF transcript throughout neurogenesis, which does not preclude the expression of neural-specific transcripts and the acquisition of the differentiated phenotype in primary spinal neurons. In contrast, conditional expression of the full-length REST/NRSF protein in PC12 cells inhibits the neuronal differentiation normally triggered by nerve growth factor in this cell line (Ballas et al., 2001). Furthermore, we observed that the expression of xREST-DBD associates with a marked repression of three different neural genes in primary spinal neurons of Xenopus embryos, and we did not observe ectopic expression of the studied genes. The results obtained by the injection of morpholino-modified antisense oligonucleotides targeted against xREST/NRSF support the possibility that the repression is a consequence of decreased activity of the endogenous protein.

Because these results contrast with those obtained in mice embryos bearing the homozygous deletion of the REST/NRSF gene, it is necessary to emphasize that the developmental stages that we analyzed are comparable with murine embryonic day (E) 7.5-8 and that studies of REST/NRSF expression in mouse embryos have focused on later stages (Chong et al., 1995; Schoenherr and Anderson, 1995; Chen et al., 1998). Significant abnormalities in REST/NRSF-deficient embryos are observed starting at E9.5-10, and $100 \%$ lethality is observed at E11.5. Therefore, early different roles of REST/NRSF may have been missed.

A plausible mechanism that can reconcile the well described repressor role and our observations is the differential recruitment of corepressor or coactivator complexes to the promoters that bear the RE-1 motif. This differential recruitment could depend on the cell type or developmental stage-specific expression of the activators or repressors, the differential accessibility or conformation of chromatin, which in turn may allosterically modulate the function of the transcription factor, or a combination of them. Although there are antecedents for the bifunctionality of transcription factors (Rao, 2001), a detailed biochemical analysis demonstrating this for REST/NRSF has not been communicated. Nevertheless, REST/NRSF can interact with the nuclear receptor corepressor (NCoR), and in turn recruitment of HDAC3 by NCoR has been associated to activation of transcription (Jepsen et al., 2000). Regarding the differences in expression patterns of possible components of complexes that include REST/NRSF, it must be noted that CoREST, described as a REST-associated corepressor, displays a predominantly neural expression pattern at very early stages of both murine and amphibian development (Grimes et al., 2000; De la Calle-Mustienes et al., 2002), although at later stages, non-neural expression is observed. This fact is compatible with the idea that REST-CoREST complexes may play a role in gene expression in the very early nervous system different from the role of repressors in non-neural cells at later stages.

The capability of REST/NRSF to ensemble diverse complexes able to modulate transcription may be used to restrict the expression of some genes to neurons, as well as to silence their nonneural expression. This general mechanism may account for the regulated expression of sodium channels and, thus, the onset of electrical excitability in developing neurons.

\section{REFERENCES}

Andrés ME, Burger C, Peral-Rubio MJ, Battaglioli E, Anderson ME, Grimes J, Dallman J, Ballas N, Mandel G (1999) CoREST: a functional corepressor required for regulation of neural-specific gene expression. Proc Natl Acad Sci USA 96:9873-9878.

Ballas N, Battaglioli E, Atouf F, Andrés ME, Chenoweth J, Anderson ME, Burger C, Moniwa M, Davie JR, Bowers WJ, Federoff HJ, Rose DW, Rosenfeld MG, Brehm P, Mandel G (2001) Regulation of neuronal traits by a novel transcriptional complex. Neuron 31:353-365.

Bessis A, Champtiaux N, Chatelin L, Changeux JP (1997) The neuronrestrictive silencer element: a dual enhancer/silencer crucial for patterned expression of a nicotinic receptor gene in the brain. Proc Natl Acad Sci USA 94:5906-5911.

Burger C, Ribera AB (1996) Xenopus spinal neurons express Kv2 potassium channel transcripts during embryonic development. J Neurosci 16:1412-1421.

Chen ZF, Paquette AJ, Anderson DJ (1998) NRSF/REST is required in vivo for repression of multiple neuronal target genes during embryogenesis. Nat Genet 20:136-142.

Chong JA, Tapia-Ramirez J, Kim S, Toledo-Aral JJ, Zheng Y, Boutros MC, Altshuller YM, Frohman MA, Kraner SD, Mandel G (1995) REST: a mammalian silencer protein that restricts sodium channel gene expression to neurons. Cell 80:949-957.

de la Calle-Mustienes E, Moldolell J, Gómez-Skarmeta JL (2002) The Xiro-repressed gene CoREST is expressed in Xenopus neural territories. Mech Dev 110:209-211.

Desarmenien MG, Clendening B, Spitzer NC (1993) In vivo development of voltage-dependent ionic currents in embryonic Xenopus spinal neurons. J Neurosci 13:2575-2581.

Grimes JA, Nielsen SJ, Battaglioli E, Miska EA, Speh JC, Berry DL, Atouf F, Holdener BC, Mandel G, Kouzarides T (2000) The corepressor mSin3A is a functional component of the REST-CoREST repressor complex. J Biol Chem 275:9461-9467.

Gurantz D, Ribera AB, Spitzer NC (1996) Temporal regulation of Shaker- and Shab-like potassium channel gene expression in single embryonic spinal neurons during $\mathrm{K}+$ current development. J Neurosci 16:3287-3295.

Hartenstein V (1989) Early neurogenesis in Xenopus: the spatiotemporal pattern of proliferation and cell lineages in the embryonic spinal cord. Neuron 3:399-411.

Huang Y, Myers SJ, Dingledine R (1999) Transcriptional repression by 
REST: recruitment of $\operatorname{Sin} 3 \mathrm{~A}$ and histone deacetylase to neuronal genes. Nat Neurosci 2:867-872.

Jepsen K, Hermanson O, Onami TM, Gleiberman AS, Lunyak V, McEvilly RJ, Kurokawa R, Kumar V, Liu F, Seto E, Hedrick SM, Mandel G, Glass CK, Rose DW, Rosenfeld MG (2000) Combinatorial roles of the nuclear receptor corepressor in transcription and development. Cell 102:753-763.

Kallunki P, Edelman GM, Jones FS (1998) The neural restrictive silencer element can act as both a repressor and enhancer of L1 cell adhesion molecule gene expression during postnatal development. Proc Natl Acad Sci USA 95:3233-3238.

Kraner SD, Chong JA, Tsay HJ, Mandel G (1992) Silencing the type II sodium channel gene: a model for neural-specific gene regulation. Neuron 9:37-44.

Kuwahara K, Saito Y, Ogawa E, Takahashi N, Nakagawa Y, Naruse Y, Harada M, Hamanaka I, Izumi T, Miyamoto Y, Kishimoto I, Kawakami R, Nakanishi M, Mori N, Nakao K (2001) The neuronrestrictive silencer element-neuron-restrictive silencer factor system regulates basal and endothelin 1-inducible atrial natriuretic peptide gene expression in ventricular myocytes. Mol Cell Biol 21:2085-2097.

Mori N, Schoenherr C, Vandenbergh DJ, Anderson DJ (1992) A common silencer element in the SCG10 and type II Na+ channel genes binds a factor present in nonneuronal cells but not in neuronal cells. Neuron 9:45-54.

Naruse Y, Aoki T, Kojima T, Mori N (1999) Neural restrictive silencer factor recruits $\mathrm{mSin} 3$ and histone deacetylase complex to repress neuron-specific target genes. Proc Natl Acad Sci USA 96:13691-13696.

O’Dowd DK, Ribera AB, Spitzer NC (1988) Development of voltagedependent calcium, sodium, and potassium currents in Xenopus spinal neurons. J Neurosci 8:792-805.

Olson EC (1996) Onset of electrical excitability during a period of circus plasma membrane movements in differentiating Xenopus neurons. J Neurosci 16:5117-5129.

Palm K, Belluardo N, Metsis M, Timmusk T (1998) Neuronal expression of zinc finger transcription factor $R E S T / N R S F / X B R$ gene. J Neurosci 18:1280-1296.

Paquette AJ, Perez SE, Anderson DJ (2000) Constitutive expression of the neuron-restrictive silencer factor (NRSF)/REST in differentiating neurons disrupts neuronal gene expression and causes axon pathfinding errors in vivo. Proc Natl Acad Sci USA 97:12318-12323.

Rao A (2001) New functions for DNA binding domains. http://stke. sciencemag.org/cgi/content/full/sigtrans;2001/81/pe1.

Ribera AB (1990) A potassium channel gene is expressed at neural induction. Neuron 5:691-701.

Ribera AB, Spitzer NC (1989) A critical period of transcription required for differentiation of the action potential of spinal neurons. Neuron 2:1055-1062.

Schoenherr CJ, Anderson DJ (1995) The neuron-restrictive silencer factor (NRSF): a coordinate repressor of multiple neuron-specific genes. Science 267:1360-1363.

Schoenherr CJ, Paquette AJ, Anderson DJ (1996) Identification of potential target genes for the neuron-restrictive silencer factor. Proc Natl Acad Sci USA 93:9881-9886.

Scholl T, Stevens MB, Mahanta S, Strominger JL (1996) A zinc finger protein that represses transcription of the human MHC class II gene, DPA. J Immunol 156:1448-1457.

Seth KA, Majzoub JA (2001) Repressor element silencing transcription factor/neuron-restrictive silencing factor (REST/NRSF) can act as an enhancer as well as a repressor of corticotropin-releasing hormone gene transcription. J Biol Chem 276:13917-13923.

Sive HL, Grainger RM, Harland RM (2000) Early development of Xenopus laevis: a laboratory manual. Cold Spring Harbor, NY: Cold Spring Harbor Laboratory.

Spitzer NC, Lamborghini JE (1976) The development of the action potential mechanism of amphibian neurons isolated in culture. Proc Natl Acad Sci USA 73:1641-1645.

Vincent A, Lautermilch NJ, Spitzer NC (2000) Antisense suppression of potassium channel expression demonstrates its role in maturation of the action potential. J Neurosci 20:6087-6094. 